\title{
A Two-Stage Diagnosis Framework for Wind Turbine Gearbox Condition Monitoring
}

\author{
Prasanna Tamilselvan ${ }^{1}$, Pingfeng Wang ${ }^{2,}{ }^{*}$, Shuangwen Sheng ${ }^{3}$, and Janet M. Twomey ${ }^{4}$ \\ ${ }^{1,2,4}$ Department of Industrial and Manufacturing Engineering, Wichita State University, Wichita, KS 67208, United States \\ pxtamilselvan@wichita.edu; \\ pingfeng.wang@wichita.edu; \\ janet.twomey@wichita.edu; \\ ${ }^{3}$ National Renewable Energy Laboratory, Golden, CO 80401, United States \\ shuangwen.sheng@nrel.gov
}

\begin{abstract}
Advances in high-performance sensing technologies enable the development of wind turbine condition monitoring systems to diagnose and predict the system-wide effects of failure events. This paper presents a vibration-based twostage fault detection framework for failure diagnosis of rotating components in wind turbines. The proposed framework integrates an analytical defect detection method with a graphical verification method to ensure diagnosis efficiency and accuracy. The efficacy of the proposed methodology is demonstrated with a case study using the gearbox condition monitoring Round Robin study dataset provided by the National Renewable Energy Laboratory (NREL). The developed methodology successfully detected five faults out of a total of seven with accurate severity levels and without producing any false alarm in the blind analysis. The case study results indicate that the developed fault detection framework is effective for analyzing gear and bearing faults in wind turbine drivetrain systems based on system vibration characteristics.
\end{abstract}

\section{INTRODUCTION}

Maintaining wind turbines in top operating condition ensures not only continuous revenue generation but a reduction in electric power drawn from non-renewable and more polluting sources. Despite the large capital cost of establishing a wind farm, the operation cost of wind turbines is one of the primary contributors to wind energy costs (Ebeling, 1997; Tamilselvan, Wang, \& Twomey, 2012; Tamilselvan, Wang, \& Wang, 2012; Tamilselvan \& Wang,

\footnotetext{
Tamilselvan, et al. This is an open-access article distributed under the terms of the Creative Commons Attribution 3.0 United States License, which permits unrestricted use, distribution, and reproduction in any medium, provided the original author and source are credited.

* Corresponding Author. Tel: +1 316978 5910; Fax: +1 3169783742.
}

2013). Unexpected breakdowns can be prohibitively expensive, as they immediately result in lost production and poor customer satisfaction. The need is becoming critical for effective wind turbine condition monitoring (CM) systems that enable accurate early-stage failure diagnosis to facilitate optimum maintenance planning. Advances in highperformance sensing and signal-processing technologies enable the development of wind turbine health monitoring systems and failure diagnosis tools that can be applied to detect, diagnose, and predict the system-wide effects of wind turbine failure events.

Maintenance activities for wind turbines can be broadly classified into two categories, namely, corrective maintenance and preventive maintenance. Corrective maintenance is carried out after a failure event, whereas preventive maintenance is done before the occurrence of a potential failure (Nielsen \& Sorensen, 2010). Preventive maintenance can be further classified into scheduled maintenance and condition-based maintenance (CBM). Scheduled maintenance can be minor or major and is carried out at fixed scheduled times. Some examples of minor scheduled maintenance for wind turbines include change of filters, lubrication, etc. (Nilsson \& Bertling, 2007). CBM is a form of preventive maintenance that involves continuous health monitoring of a wind turbine unit. Currently, the most common maintenance practice for wind farms is scheduled maintenance. However, with recent developments in the field of sensing and signal-processing techniques, CBM has been gradually adopted into maintenance decision making for wind farms (Byon, Perez, Ntaimo, \& Ding, 2010). In CBM, condition monitoring systems are installed on different system components, such as the gearbox, bearings, drivetrain, and generators, to record various sensory signals in order to determine the physical states of these components. Different types of sensory signals can be used for CM purposes, such as vibration and electrical 
signals. Usually, CBM of wind turbines can be executed based on vibration monitoring (Shi, Wang, Zhuo, \& Liu, 2010; Gebraeel, Lawley, \& Liu, 2002; Randall, 2011; Randall \& Antoni, 2011; McFadden \& Smith, 1984; Antoni, 2002; Harris, 2001; Lebold, McClintic, Campbell, Byington, \& Maynard, 2000), oil analysis (Lu \& Chu, 2010), or electrical signature analysis (Yang, Tavner, \& Wilkinson, 2008). With the help of wind turbine health information provided by CM systems, optimal operation and maintenance (O\&M) planning strategies can be ascertained to prevent system failures and improve wind turbine availability.

Vibration analysis is one of the most commonly used mechanisms for CM of wind turbines (Shi, Wang, Zhuo, \& Liu, 2010). It is mainly utilized to identify the present condition of wind turbine components, such as the gearbox, drivetrain, bearings, and so on, and estimate their damage growth over time. In vibration analysis, vibration signals produced by the rotating wind turbine components whose current health conditions need to be diagnosed are commonly analyzed either by broadband-based methods or spectral line analysis methods ( $\mathrm{Lu} \& \mathrm{Chu}, 2010)$. In broadband analysis, parameters such as root mean square, peak value, or kurtosis are calculated based on the obtained output signals. Component failure can be estimated by the changes observed in the values of the above calculated parameters. Spectral line analysis is based on the theory that each component exhibits different vibration signatures frequencies. These frequencies vary for each component, such as the gear mesh, shaft harmonics, or bearing harmonics. Component failure is said to occur if there is a measurable increase in the frequency of the impulse signals for individual components.

Research on real-time failure diagnosis, which interprets data acquired by smart sensors and utilizes these data streams in making critical decisions, provides significant advancements for wind turbine fault detection so that the health condition of a wind turbine can be determined before unexpected failures are developed (Tamilselvan, Wang, \& Jayaraman, 2012; Tamilselvan \& Wang, 2012; Tamilselvan \& Wang, 2013; Byon et al., 2010). Among the many mechanisms for wind turbine CM, one of the most vastly used is vibration-based health monitoring systems, which detect wind turbine component faults based on the vibration signals produced by the rotating components during operation. Although effective health diagnosis for wind turbines provides various benefits, such as improved reliability and reduced turbine maintenance costs, analysis of massive heterogeneous vibration signals leading to accurate early-stage detection of wind turbine component failure remains a challenge.

This paper presents a vibration-based two-stage fault detection framework and integrates an analytical defect detection method with a graphical verification method to ensure efficient and accurate failure diagnosis. The proposed methodology is demonstrated with NREL's wind turbine gearbox CM Round Robin study, and the results are discussed. The rest of the paper is organized as follows. Section 2 presents the proposed vibration-based CM framework. Section 3 introduces the Round Robin gearbox CM experiment, and Section 4 details the data preprocessing. The analytical diagnosis method and the graphical verification method are detailed in Sections 5 and 6 , respectively. Section 7 reports the CM Round Robin study results, and Section 8 briefly summarizes the work.

\section{VibRATION-BASED HEALTH DIAGNOSIS FRAMEWORK}

The framework for the proposed vibration-based two-stage health diagnosis is shown in Figure 1. The developed framework is composed of three essential modules: (i) data preprocessing for conversion of time domain vibration signals to frequency domain signals; (ii) an analytical diagnosis module for the detection of defects in the rotating components using sideband and kurtosis evaluation, as shown in the bottom left shaded box, which includes the severity factor and matrix determination process; and (iii) a graphical diagnosis module to determine the level of severity of the defect.

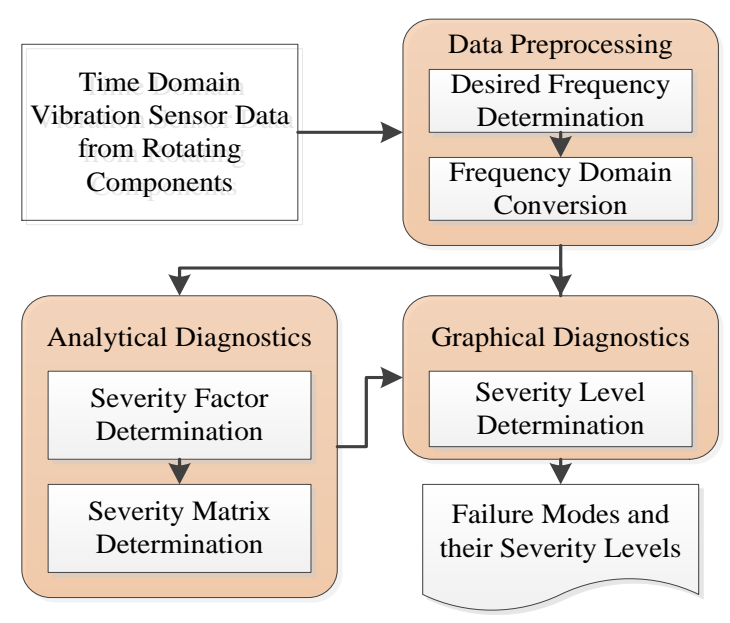

Figure 1. Vibration-based two-stage health diagnosis framework

The time domain vibration sensory signals from the rotating components are preprocessed and converted into frequency domain signals for further CM analysis. The sideband- and kurtosis-based detection method is employed to analyze the frequency data to detect failures of the rotating components. The results of the analytical diagnosis are used as inputs to the graphical diagnosis process. The failure modes and their severity levels are determined from the frequency domain signals by graphical diagnosis.

\subsection{Vibration Data Preprocessing}


The preprocessing of vibration data involves three steps, as shown in Table 1. The first step of the vibration analysis is to calculate the desired frequencies for the rotating components. For instance, the gear meshing frequencies (GMF) and shaft rotation frequency (SRF) are desired frequencies for gears, and ball passing frequency in outer race (BPFO), ball passing frequency in inner race (BPFI), and ball spinning frequency (BSF) are desired frequencies for bearings. The next step involves the identification of the relationship between the sensors and the components. Based on the identified relationship, the sensors related to different failure modes are segregated, and their corresponding desired frequencies are noted. The final preprocessing step is to develop the frequency spectrum from the raw time domain vibration signal using the fast Fourier transformation (FFT) process for the desired sensors.

\begin{tabular}{l|l}
\hline Step 1 & \multicolumn{2}{|l}{ Calculate GMF for gears and bearing frequencies } \\
\hline Step 2 & $\begin{array}{l}\text { Determine relationship between sensors and } \\
\text { components }\end{array}$ \\
\hline Step 3 & Develop FFT plot for desired sensors in each case \\
\hline
\end{tabular}

Table 1. Procedure for vibration data preprocessing

\subsection{Analytical Diagnosis}

The online analytical diagnosis approach identifies defects or failures in the rotating components from the preprocessed frequency domain data. The developed analytical diagnosis method helps to narrow down the whole frequency spectrum to potential failure modes and their frequencies. The developed method with sideband- and kurtosis-based online defect detection processes the frequency data analytically; the stepwise procedure is shown in Table 2 .

The maximum amplitude values for the desired frequencies, the sidebands, and the kurtosis values for the sidebands are determined to calculate the severity factors to formulate the defect severity matrix. The failure modes and their severity levels from the frequency domain signals are determined by the defect severity matrix. The results from the analytical diagnosis are given as inputs to the graphical diagnosis process.

\begin{tabular}{c|l}
\hline Step 1 & $\begin{array}{l}\text { Determine maximum amplitude values for } \\
\text { sidebands and desired frequency }\end{array}$ \\
\hline Step 2 & Determine kurtosis values for sidebands \\
\hline Step 3 & Calculate severity factors 1, 2, and 3 \\
\hline Step 4 & Formulate defect severity matrix \\
\hline
\end{tabular}

Table 2. The analytical diagnosis procedure

\subsubsection{Sideband and Kurtosis Analysis}

The sidebands are indicators of the failure modes in the frequency spectrum of each rotating component based on their spread on both sides of the desired frequency. The rising and inequality of the sidebands correspond to component defects; moreover, the severity of these defects can be identified based on the frequency sideband features, as listed in Table 3.

The height and sharpness of the peak amplitudes in each frequency spectrum are measured by kurtosis. The spread of the sidebands on either side of the desired frequency can be analyzed using kurtosis values. Differences in kurtosis values for both sidebands denote their inequality. The kurtosis ratio, $K R$, is the ratio of the left side of the $j^{\text {th }}$ frequency spectrum, where the desired frequency is $K_{L j}$, to the right side of the $j^{\text {th }}$, where the desired frequency is $K_{R j}$, as shown in Eq. 1. Similarly, the ratio of maximum amplitude of the sideband on the left and right sides of the $j^{\text {th }}$ frequency is determined as $A R$, as shown in Eq. 1.

$$
K R=\frac{K_{L j}}{K_{R j}} ; A R=\frac{A_{L j}}{A_{R j}}
$$

\begin{tabular}{l|c}
\hline Frequency Sideband Feature & Severity Level \\
\hline $\begin{array}{l}\text { Rising of sidebands around } \\
\text { desired frequency }\end{array}$ & Low \\
\hline $\begin{array}{l}\text { Unequal sidebands on both } \\
\text { sides }\end{array}$ & Medium \\
\hline $\begin{array}{l}\text { Higher sideband amplitude than } \\
\text { frequency amplitude }\end{array}$ & High \\
\hline
\end{tabular}

Table 3. Sideband-based damage severity definition (SpectraQuest, 2006)

\subsubsection{Severity Factors}

The different failure modes and their severity levels are determined from the converted frequency domain signal through sideband and kurtosis analysis. Table 3 shows the different severity levels based on the frequency sideband features. The three severity factor metrics developed for online defect detection are as follows.

Severity factor $1\left(S F_{1}\right)$ ensures equal spread of the sidebands using the kurtosis ratio metric, as shown in Eq. 2. The threshold kurtosis ratio, $K R_{T}$, is considered to be 0.6 . The value of $S F_{1}<1$ denotes the unequal spread of sidebands and vice versa. Severity factor $2\left(S F_{2}\right)$ ensures equal maximum amplitude of the sidebands on both sides of the desired frequency, as shown in Eq. 3. The threshold amplitude ratio, $A_{T}$, is considered to be 0.9 . The value of $S F_{2}$ $<1$ denotes the unequal frequency amplitudes on both sides of the sidebands and vice versa.

$$
\begin{aligned}
& S F_{1}=\frac{\operatorname{Min}\left(K R_{j}, K R_{j}^{-1}\right)}{K R_{T}} \\
& S F_{2}=\frac{\operatorname{Min}\left(A R_{j}, A R_{j}^{-1}\right)}{A_{T}}
\end{aligned}
$$

Severity factor $3\left(\mathrm{SF}_{3}\right)$ ensures that the maximum desired 
frequency amplitude is higher than the maximum amplitude of the sideband, $A_{\max }$, as shown in Eq. 4, where $A_{F}$ is the maximum amplitude at the desired frequency. The value of $\mathrm{SF}_{3}<1$ denotes the frequency amplitude of the sideband, $A_{\text {max }}$, which is higher than the desired frequency, $A_{F}$.

$$
S F_{3}=\frac{A_{F}}{\operatorname{Max}\left(A_{L j}, A_{R j}\right)}
$$

The conditions $S F_{1} \leq 1, S F_{2}>1$, and $S F_{3}>1$ show that the component has a low (L)-severity defect. The severity factor characteristics of the medium (M)-severity defect are: $S F_{1} \leq$ $1, S F_{2} \leq 1$, and $S F_{3}>1$; and $S F_{1}>1, S F_{2} \leq 1$, and $S F_{3}>1$. Similarly, the high $(\mathrm{H})$-severity defect conditions are: $\mathrm{SF}_{1} \leq$ $1, S F_{2} \leq 1$, and $S F_{3} \leq 1 ; S F_{1}>1, S F_{2} \leq 1$, and $S F_{3} \leq 1 ; S F_{1} \leq$ $1, S F_{2}>1$, and $S F_{3} \leq 1$; and $S F_{1}>1, S F_{2}>1$, and $S F_{3} \leq 1$. If all three severity factors are greater than one, then the component has no defect $(\mathrm{N})$. Based on these rules, the severity levels and the failure modes of the components are identified based on each sensor. The procedure for assigning severity levels is clearly shown as a flowchart in Figure 2. The determined severity level will be assigned to the corresponding component $u$ at level $g$ through sensor $m$ $\left(S_{\text {ugm }}\right)$. Similarly, the FFTs of different sensors are analyzed, and $S_{u g m}$ values are determined for all desired components.

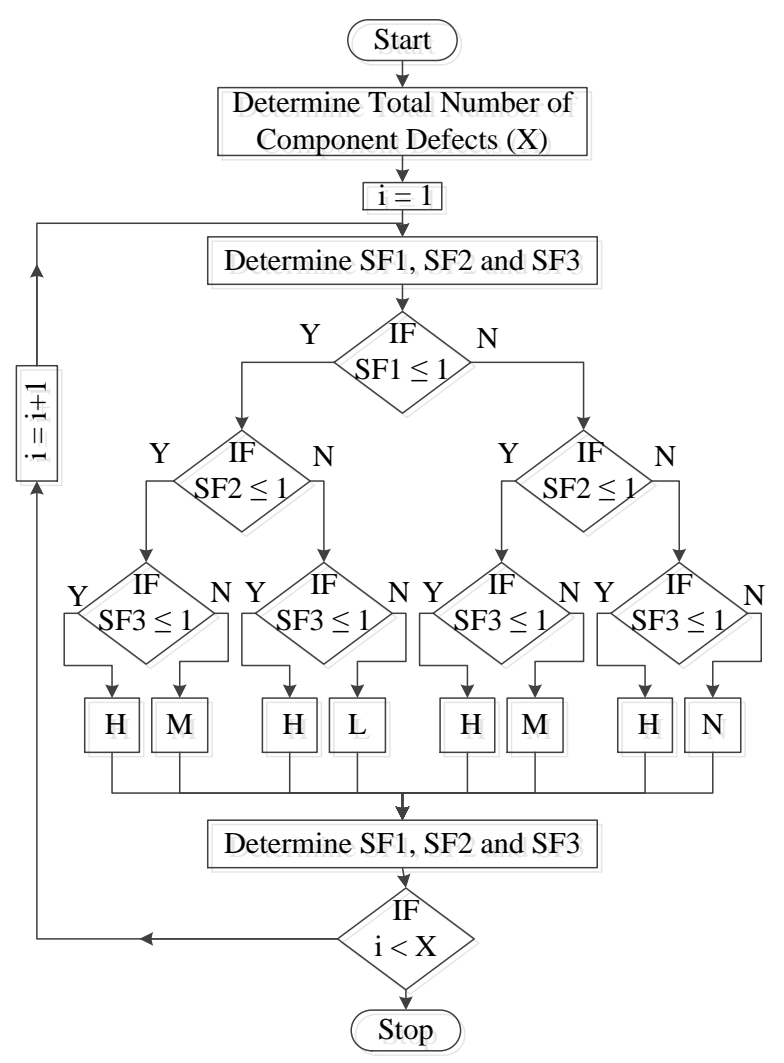

Figure 2. Flowchart of the severity-level assigning procedure

\subsubsection{Severity Defect Matrix}

The failure modes and the severity levels of the rotating components based on each sensor are identified with different severity metrics. However, the same defect of a rotating component can be identified by different sensors in and around the component location. Therefore, there is a need to develop a unified metric to make decisions on the failure mode and its severity level. The combination of results for all the components from the different sensors leads to the development of a defect severity matrix. The desired component matrix $T$ is shown in Eq. 5.

$$
T=\left(\begin{array}{c}
\text { Desired Component } 1 \\
\text { Desired Component } 2 \\
\vdots \\
\text { Desired Component } U
\end{array}\right)
$$

The desired component matrix and the severity factor levels of all the components are utilized to develop a defect severity matrix. The severity ratio of component $u$ at severity level $g, S_{u g}$, is represented as Eq. 6, where $g$ represents the different severity levels (i.e., low, medium, high), and $S_{u g m}$ represents the severity level of component $u$ at level $g$ through sensor $m$.

$$
S_{u g}=\sum_{m=1}^{M} S_{u g m} / \sum_{g=1}^{3} \sum_{m=1}^{M} S_{u g m}
$$

The defect severity matrix, $D S$, represents the defect component and its severity level in the matrix format as shown in Eq. 7, where rows of the matrix represent each desired component, and columns represent the severity level of the components (i.e., low, medium, high). The analytical results are further fine-tuned using the graphical diagnosis process.

$$
D S=\left(\begin{array}{ccc}
S_{11} & S_{12} & S_{13} \\
\vdots & \ddots & \vdots \\
S_{U 1} & S_{U 2} & S_{U 3}
\end{array}\right)
$$

\subsection{Graphical Diagnosis}

The unified defect severity matrix results provide initial insights about the component defects and their severity levels. However, false identifications are possible in the analytical methodology due to the overlap of different frequencies and their harmonic levels. Therefore, there is a need to verify identified component defects graphically. The frequency spectra of the predetermined component defects are verified graphically based on the sideband amplitudes and their spread. The developed two-stage fault detection framework is demonstrated with NREL's Round Robin gearbox CM study in the next section. 


\section{GEARBOX DIAGNOSIS ROUND ROBIN STUDY}

The NREL Round Robin gearbox reliability collaborative (GRC) test turbine drivetrain (Sheng et al., 2011; Sheng, 2012) is shown in Figure 3. The time domain vibration signals from the sensors placed on the gearbox are used for $\mathrm{CM}$ to determine the defects in the gears and bearings of the GRC test gearbox.

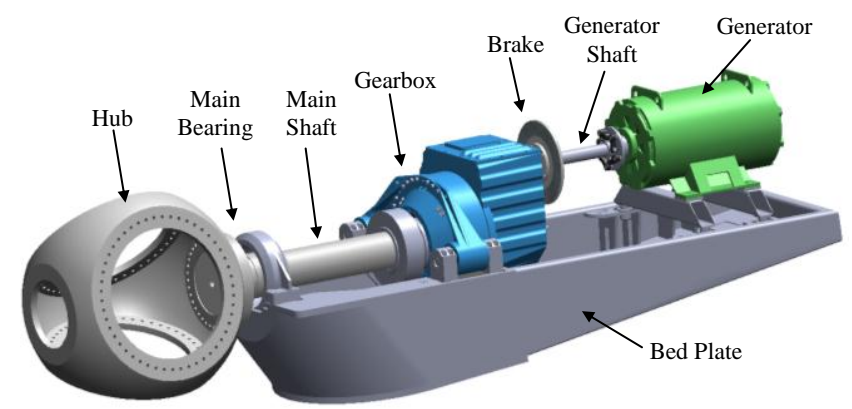

Figure 3. GRC test turbine drivetrain (Sheng, 2012)

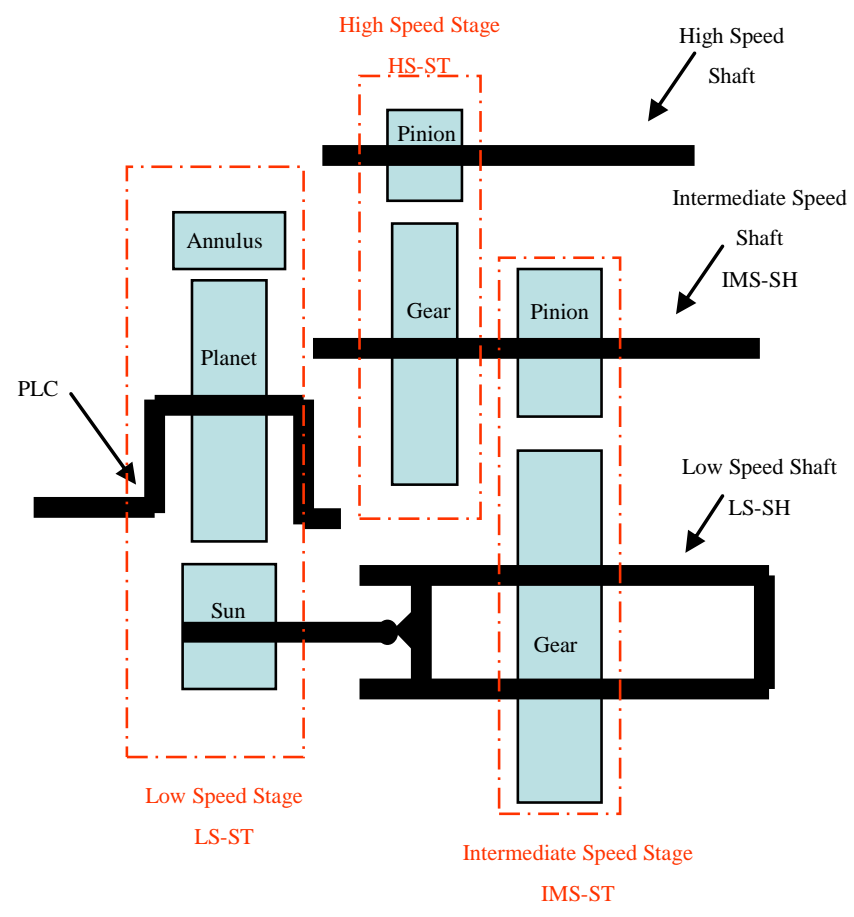

Figure 4. GRC gearbox internal nomenclature and abbreviations (Sheng, 2012)

The GRC gearbox has an overall gear ratio of 1:81.491. It has one planetary stage and two parallel stages, namely, a high-speed stage (HS-ST) and an intermediate-speed stage (IS-ST), as shown in Figure 4. The main shaft is connected to the planetary arm of the gearbox, and the high speed pinion of the gearbox is geared to the generator. The experiment was conducted at two speed levels: $1200 \mathrm{rpm}$ and $1800 \mathrm{rpm}$.

\begin{tabular}{c|c|c|c}
\hline Test Case & $\begin{array}{c}\text { Electric } \\
\text { Power } \\
(\% \text { of rated })\end{array}$ & $\begin{array}{c}\text { Duration } \\
(\mathrm{min})\end{array}$ & $\begin{array}{c}\text { Speed } \\
(\mathrm{rpm})\end{array}$ \\
\hline CM_2a & $25 \%$ & 10 & 1200 \\
\hline CM_2b & $25 \%$ & 10 & 1800 \\
\hline CM_2c & $50 \%$ & 10 & 1800 \\
\hline
\end{tabular}

Table 4. Test case data description

The three test cases were conducted at different power ratings and speed levels, as shown in Table 4. Data for each sensor placed on the gearbox were collected as ten 1-minute datasets for each test case.

\begin{tabular}{c|l|l}
\hline Damage \# & $\begin{array}{c}\text { Component / } \\
\text { Location }\end{array}$ & \multicolumn{1}{c}{ Mode } \\
\hline $\mathbf{1}$ & HSS gear set & Scuffing \\
\hline $\mathbf{2}$ & $\begin{array}{l}\text { HSS downwind } \\
\text { bearing (DWB) }\end{array}$ & Overheating \\
\hline $\mathbf{3}$ & ISS gear set & $\begin{array}{l}\text { Fretting corrosion, } \\
\text { scuffing, polishing } \\
\text { wear }\end{array}$ \\
\hline $\mathbf{5}$ & $\begin{array}{l}\text { ISS upwind } \\
\text { bearing (UWB) }\end{array}$ & $\begin{array}{l}\text { Assembly damage, } \\
\text { plastic deformation, } \\
\text { scuffing, contact } \\
\text { corrosion }\end{array}$ \\
\hline $\mathbf{6}$ & $\begin{array}{l}\text { ISS DWB* } \\
\text { gear or sun } \\
\text { pinion }\end{array}$ & $\begin{array}{l}\text { Assembly damage, } \\
\text { plastic deformation, } \\
\text { dents }\end{array}$ \\
\hline $\mathbf{7}$ & $\begin{array}{l}\text { Planet carrier } \\
\text { UWB** }\end{array}$ & $\begin{array}{l}\text { Scuffing, polishing } \\
\text { and fretting corrosion }\end{array}$ \\
\hline \\
* Damage was on the spacer, not on the bearing corrosion \\
** Damage was only on the non-rolling surfaces of the bearing
\end{tabular}

Table 5. Actual damages in the wind turbine gearbox (Sheng, 2012)

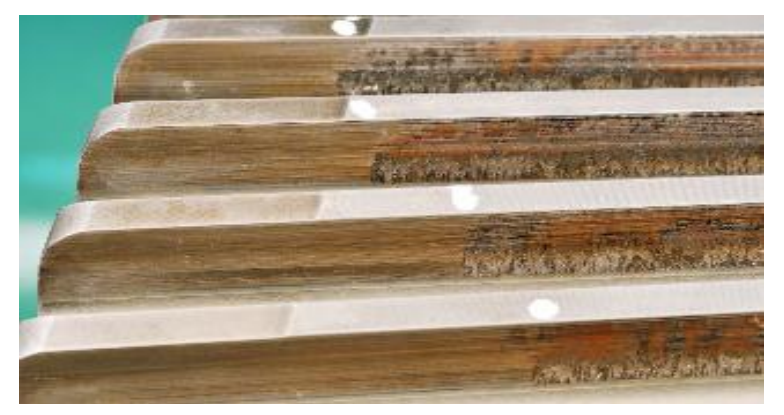

Figure 5. Test gearbox high-speed-stage gear damage (Sheng, 2012)

Gearbox CM was carried out by mounting 12 sensors at various locations around the drivetrain. The vibration data was collected at $40 \mathrm{kHz}$ per channel using a National 
Instruments PXI -4472B high-speed data acquisition system (DAQ); further details about the experimental setup can be found in Sheng (2012). NREL provided the real damage results for the GRC gearbox (Errichello \& Muller, 2012), and those damages selected for vibration analysis algorithm performance evaluation in the Round Robin study are listed in Table 5, in which HSS denotes the high-speed shaft, ISS denotes the intermediate-speed shaft, and LSS denotes the low-speed shaft. Figure 5 shows the real damage on highspeed gear.

\section{Data Preprocessing}

The Round Robin study involves three speed stages: low speed (LS-ST), intermediate speed (IS-ST), and high speed (HS-ST). Among the total sensors from the Round Robin GRC gearbox, the desired sensors for the analysis of the LSST, IS-ST, and HS-ST are AN3 and AN5 to AN10. The relationships between these sensors and the components are determined based on sensor location and proximity to the rotating components as listed in Table 6. The damages which are capable of being detected by each sensor are also listed.

\begin{tabular}{|c|c|c|c|c|}
\hline $\begin{array}{l}\text { Sensor } \\
\text { Name }\end{array}$ & Gear & Shaft & Bearing & Damage \\
\hline $\begin{array}{l}\text { AN3- } \\
\text { planet } \\
\text { radial } \\
180\end{array}$ & $\begin{array}{l}\text { Planet } \\
\text { gear \& } \\
\text { sun } \\
\text { pinion }\end{array}$ & $\begin{array}{l}\text { Planet } \\
\text { arm }\end{array}$ & $\begin{array}{l}\text { Planet } \\
\text { carrier } \\
\text { UWB and } \\
\text { DWB }\end{array}$ & $\begin{array}{l}\text { Planet gear } \\
\text { defect, planetary } \\
\text { arm bearing } \\
\text { defect, planet } \\
\text { bearing defect }\end{array}$ \\
\hline $\begin{array}{l}\text { AN5- } \\
\text { LSS } \\
\text { radial }\end{array}$ & $\begin{array}{l}\text { IS-ST } \\
\text { gear }\end{array}$ & LSS & $\begin{array}{l}\text { LSS } \\
\text { UWB and } \\
\text { DWB }\end{array}$ & $\begin{array}{l}\text { LSS bearings } \\
\text { defect, ISS gear } \\
\text { defect }\end{array}$ \\
\hline $\begin{array}{l}\text { AN6- } \\
\text { ISS } \\
\text { radial }\end{array}$ & $\begin{array}{l}\text { IS-ST } \\
\text { pinion \& } \\
\text { HS gear }\end{array}$ & ISS & ISS & $\begin{array}{l}\text { ISS bearings } \\
\text { defect, IS pinion } \\
\text { defect, HS gear } \\
\text { defect }\end{array}$ \\
\hline $\begin{array}{l}\text { AN7- } \\
\text { HSS } \\
\text { radial }\end{array}$ & $\begin{array}{l}\text { HS-ST } \\
\text { pinion }\end{array}$ & HSS & HSS & $\begin{array}{l}\text { HSS bearings } \\
\text { defect, HS pinion } \\
\text { defect }\end{array}$ \\
\hline $\begin{array}{l}\text { AN8- } \\
\text { HSS } \\
\text { radial }\end{array}$ & $\begin{array}{l}\text { HS-ST } \\
\text { pinion }\end{array}$ & HSS & $\begin{array}{l}\text { HSS } \\
\text { UWB }\end{array}$ & $\begin{array}{l}\text { HS pinion defect, } \\
\text { HSS UWB } \\
\text { defect }\end{array}$ \\
\hline $\begin{array}{l}\text { AN9- } \\
\text { HSS rear } \\
\text { radial }\end{array}$ & $\begin{array}{l}\text { HS-ST } \\
\text { pinion }\end{array}$ & HSS & $\begin{array}{l}\text { HSS } \\
\text { DWB }\end{array}$ & $\begin{array}{l}\text { HS pinion defect, } \\
\text { HSS DWB } \\
\text { defect }\end{array}$ \\
\hline $\begin{array}{l}\text { AN10- } \\
\text { carrier } \\
\text { rear } \\
\text { radial }\end{array}$ & $\begin{array}{l}\text { Planet } \\
\text { gear \& } \\
\text { sun } \\
\text { pinion }\end{array}$ & $\begin{array}{l}\text { Planet } \\
\text { arm }\end{array}$ & $\begin{array}{l}\text { Planet } \\
\text { UWB and } \\
\text { DWB }\end{array}$ & $\begin{array}{l}\text { Planet gear } \\
\text { defect, planetary } \\
\text { arm bearing } \\
\text { defect }\end{array}$ \\
\hline
\end{tabular}

Table 6. Sensor and component relationship

\begin{tabular}{|c|c|c|c|c|}
\hline $\begin{array}{l}\text { Gear } \\
\text { Element }\end{array}$ & $\begin{array}{c}\text { \# of } \\
\text { Teeth }\end{array}$ & $\begin{array}{c}\text { Speed } \\
(\mathrm{rpm})\end{array}$ & $\begin{array}{c}\text { GMF } \\
(\mathrm{Hz})\end{array}$ & $\begin{array}{l}\text { SRF } \\
(\mathrm{Hz})\end{array}$ \\
\hline Ring gear & 99 & Fixed & NA & NA \\
\hline Planet gear & 39 & 14.74 & \multirow{2}{*}{29.45} & 0.25 \\
\hline Sun pinion & 21 & \multirow{2}{*}{84.15} & & \multirow{2}{*}{1.4025} \\
\hline LSS gear & 82 & & \multirow[b]{2}{*}{115} & \\
\hline $\begin{array}{l}\text { Intermediate } \\
\text { pinion }\end{array}$ & 23 & \multirow[t]{2}{*}{300} & & \multirow[t]{2}{*}{5} \\
\hline ISS gear & 88 & & \multirow{2}{*}{440} & \\
\hline HSS pinion & 22 & 1200 & & 20 \\
\hline
\end{tabular}

Table 7. Desired gear frequencies at $1200 \mathrm{rpm}$

\begin{tabular}{|c|c|c|c|c|c|}
\hline Location & Type & $\begin{array}{c}\text { Speed } \\
(\mathrm{rpm})\end{array}$ & $\begin{array}{c}\text { BPFI } \\
(\mathrm{Hz})\end{array}$ & $\begin{array}{c}\text { BPFO } \\
(\mathrm{Hz})\end{array}$ & $\begin{array}{l}\text { BSF } \\
(\mathrm{Hz})\end{array}$ \\
\hline \multirow{2}{*}{$\begin{array}{l}\text { Planet } \\
\text { carrier }\end{array}$} & UWB & \multirow{2}{*}{14.74} & 6.11 & 5.44 & 2.11 \\
\hline & DWB & & 6.65 & 5.88 & 1.99 \\
\hline Planet & $\begin{array}{c}\text { DWB } \\
\& \\
\text { UWB }\end{array}$ & 45.31 & 7.81 & 5.78 & 2.46 \\
\hline \multirow[b]{2}{*}{ LSS } & UWB & \multirow[b]{2}{*}{84.15} & 42.19 & 37.75 & 13 \\
\hline & DWB & & 30.6 & 26.9 & $\begin{array}{c}10.1 \\
5 \\
\end{array}$ \\
\hline \multirow{2}{*}{ ISS } & UWB & \multirow{2}{*}{300} & 49 & 35.9 & 15.6 \\
\hline & DWB & & 84.6 & 70.4 & 26 \\
\hline \multirow{2}{*}{ HSS } & UWB & \multirow{2}{*}{1200} & 197 & 143 & 62.5 \\
\hline & DWB & & 228 & 172 & 66.5 \\
\hline
\end{tabular}

Table 8. Desired bearing frequencies at $1200 \mathrm{rpm}$

The desired gear and bearing frequencies are determined and listed in Tables 7 and 8, respectively, where UWB and DWB refer to the upwind bearings and downwind bearings, respectively. The defects from the bearings and gears can be identified from their corresponding desired frequency amplitudes in the frequency spectrum. FFT converts the time domain vibration signal into a frequency domain signal and helps to analyze each desired frequency based on its amplitude and its harmonics.

\section{Analytical Diagnosis}

The analytical diagnosis process identifies the bearing and gear defects in the GRC gearbox from the preprocessed frequency domain data. The developed analytical diagnosis method is used to determine the severity factors and defect matrix and confine the whole frequency spectrum into different potential failure modes. Based on the rules specified in Section 2.2 about the severity levels and the failure modes of the components, severity factors 1, 2, and 3 
for the different gears and bearings of the GRC gearbox are determined. The severity factor analysis of sensor AN6 for case $2 \mathrm{a}$ is listed in Table 9 .

\begin{tabular}{l|c|c|c|c|c}
\hline Component & $\begin{array}{c}\text { ISS } \\
\text { Gears }\end{array}$ & $\begin{array}{c}\text { ISS } \\
\text { UWB }\end{array}$ & $\begin{array}{c}\text { ISS } \\
\text { DWB }\end{array}$ & $\begin{array}{c}\text { HSS } \\
\text { UWB }\end{array}$ & $\begin{array}{c}\text { HSS } \\
\text { DWB }\end{array}$ \\
\hline $\begin{array}{l}\text { Desired } \\
\text { Frequency }\end{array}$ & GMF & BPFI & BPFO & BPFO & BPFI \\
\hline$S F_{1}$ & 0.49 & 0.98 & 0.86 & 0.58 & 0.72 \\
\hline$S F_{2}$ & 0.97 & 0.73 & 0.42 & 1.06 & 0.58 \\
\hline$S F_{3}$ & 0.46 & 2.85 & 2.94 & 0.23 & 1.67 \\
\hline Low & 0 & 0 & 0 & 0 & 0 \\
\hline Medium & 0 & 1 & 1 & 0 & 1 \\
\hline High & 1 & 0 & 0 & 1 & 0 \\
\hline
\end{tabular}

Table 9. Severity factor analysis of sensor AN6 for case 2a

$T=\left(\begin{array}{l}\text { Planet gear and sun pinion } \\ \text { Planet carrier upwind bearing } \\ \text { Planet carrier downwind bearing } \\ \text { Planet up and downwind bearing } \\ \text { ISS gear and ISS pinion } \\ \text { HSS gear and HSS pinion } \\ \text { LSS upwind bearing } \\ \text { LSS downwind bearing } \\ \text { ISS upwind bearing } \\ \text { ISS downwind bearing } \\ \text { HSS upwind bearing } \\ \text { HSS downwind bearing }\end{array}\right)$

$$
D S=\left(\begin{array}{ccc}
0 & 0 & 0 \\
0 & 0 & 0 \\
0 & 0 & 0 \\
0 & 0 & 0 \\
0.33 & 0.34 & 0.33 \\
0.50 & 0.50 & 0 \\
0 & 0 & 0 \\
0 & 0 & 0 \\
0.67 & 0.33 & 0 \\
0.50 & 0.50 & 0 \\
0.50 & 0.33 & 0.17 \\
0.60 & 0.40 & 0
\end{array}\right)
$$

The desired component matrix $T$ for the GRC gearbox is shown in Eq. 8. The unified defect severity matrix for the Round Robin study is determined using the proposed severity factors and is shown in Eq. 9. The analytical diagnosis results indicate that there are no defects in the LSS UWB and DWB. In this study, the severity level of the damage has been evaluated quantitatively based on the vibration signals, specifically the frequency sidebands. Although it falls outside the scope of this work, it will also be very interesting to investigate how different severity levels defined and measured in this study relate to the maintenance recommendations in practice in future studies. The defect severity matrix values show that the IS gear and pinion each have a high-severity defect; the HS gear and pinion each have a medium-severity defect; and the ISS UWB, ISS DWB, and HSS DWB each have a mediumseverity defect. The HSS UWB severity matrix values show that there exists a high-severity defect. These defect results are used as the input information for the graphical diagnosis process, and the analytical results are further verified and fine-tuned, as introduced in the next section.

\section{GRAPHICAL DIAGNOSIS}

The frequency spectra of the predetermined component defects are verified graphically based on the sideband amplitudes and their spread. The graphical diagnosis results are shown as frequency plots (Figures 6-10) for the identified damages.

Figure 6 shows the HSS gear GMF $(660 \mathrm{~Hz})$ and its sidebands for the AN7 HSS radial sensor. The unequal high sidebands on both sides with GMF maximum amplitude show that there is a high-severity failure in the HSS gear. Similarly, Figure 7 shows the second harmonic of the BPFO $(172 \mathrm{~Hz})$ of the HSS DWB at $(344 \mathrm{~Hz})$ for the AN6 ISS radial sensor. The $A_{\max }$ of the right sideband is almost two times the $A_{\max }$ of the left sideband; moreover, the high amplitude of the right sideband is almost eight times the high amplitude of the desired frequency. These inferences from the figure prove that there is a high-severity failure in the outer raceway. Because the sideband amplitudes are found in the second harmonic, there is a chance of misalignment of the bearing. Figure 8 shows the ISS gear GMF $(115 \mathrm{~Hz})$ and its sidebands for the AN5 LSS radial sensor. The rise of the sidebands on both sides of the GMF maximum amplitude show that the ISS gear is in an early stage of failure.

The damage of the ISS UWB is verified graphically using the frequency plot shown in Figure 9. The sensor value used is the AN6 ISS radial, and the BPFI is $53.8 \mathrm{~Hz}$. The sideband amplitudes rise around the amplitude of the BPFI in the second harmonic frequency. The frequency plot clearly shows that there is an early inner raceway failure and bearing misalignment. Similarly, Figure 10 shows the BPFO of the ISS DWB at $73.7 \mathrm{~Hz}$ for the AN6 ISS radial sensor. The sideband amplitudes rise around one side of the maximum amplitude of the BPFO. These inferences from the figure prove that there is a high-severity failure in the 
outer raceway. Thus, the component defects are identified graphically, and the results are discussed in the next section.

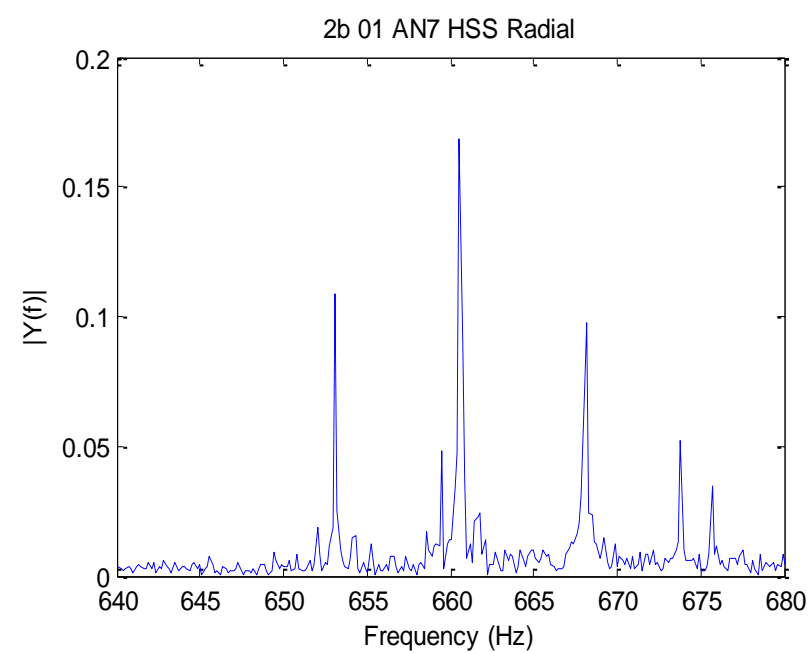

Figure 6. Damage 1, HSS pinion

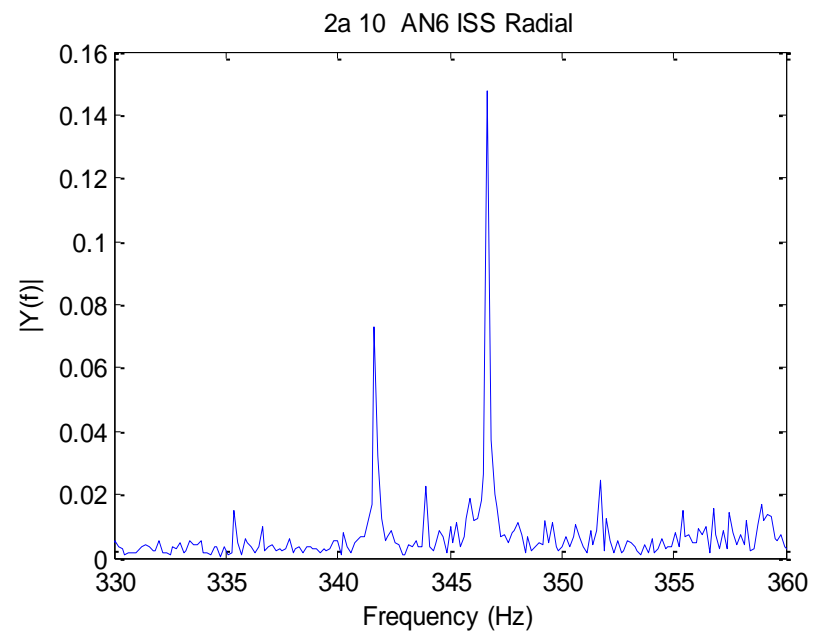

Figure 7. Damage 2, HSS DWB BPFO

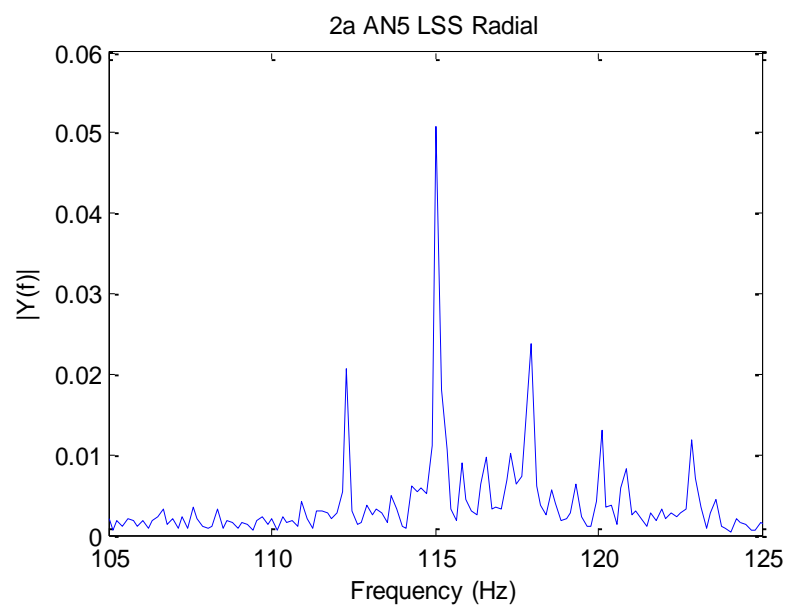

Figure 8. Damage 3, ISS gear AN5 LSS radial

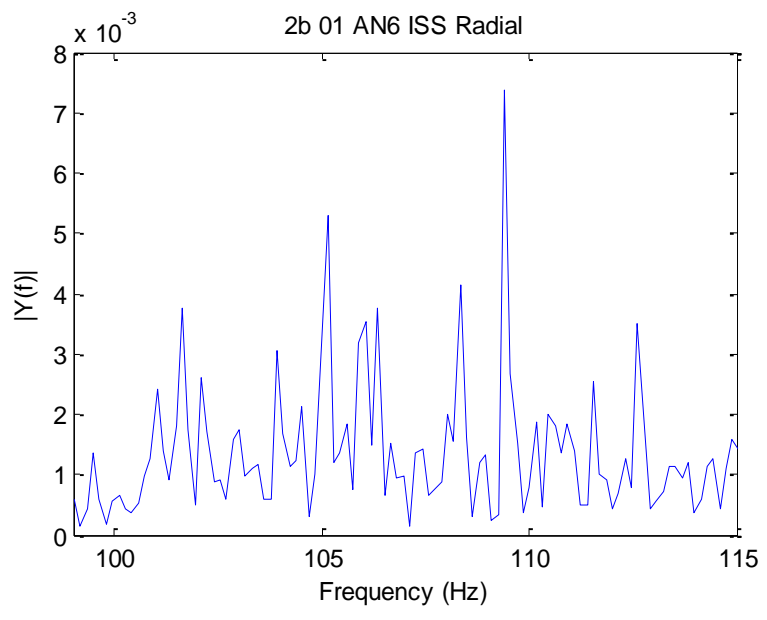

Figure 9. Damage 4, ISS UWB BPFI

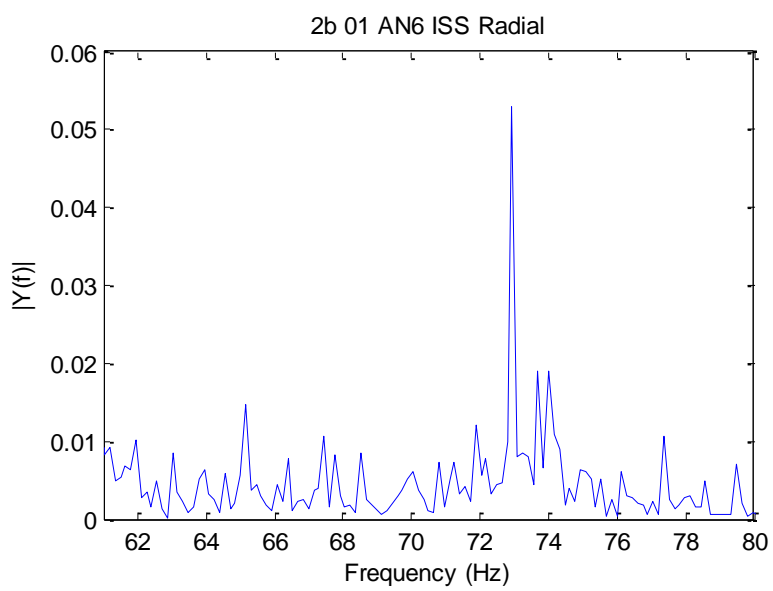

Figure 10. Damage 5, ISS DWB BPFO

\section{DiAgNOSIS RESULTS}

The results from the online analytical defect detection method are used as an input to the graphical diagnosis. The failure modes and their severity levels from the frequency domain signals are verified graphically, and the results are unified to the component level, with their corresponding severity levels, as shown in Table 10.

\begin{tabular}{c|c|c|c}
\hline Damage & Component & Mode & Severity \\
\hline $\mathbf{1}$ & HSS pinion & $\begin{array}{c}\text { Gear tooth failure } \\
\text { of HSS pinion }\end{array}$ & High \\
\hline $\mathbf{2}$ & HSS DWB & $\begin{array}{c}\text { Outer race failure } \\
\text { and bearing } \\
\text { misalignment }\end{array}$ & High \\
\hline $\mathbf{3}$ & ISS gear & $\begin{array}{c}\text { Early stages of } \\
\text { gear failure }\end{array}$ & Low \\
\hline $\mathbf{4}$ & ISS UWB & $\begin{array}{c}\text { IR failure and } \\
\text { bearing } \\
\text { misalignment }\end{array}$ & Medium \\
\hline $\mathbf{5}$ & ISS DWB & OR failure & High \\
\hline
\end{tabular}

Table 10. Gearbox fault diagnosis blind analysis results 


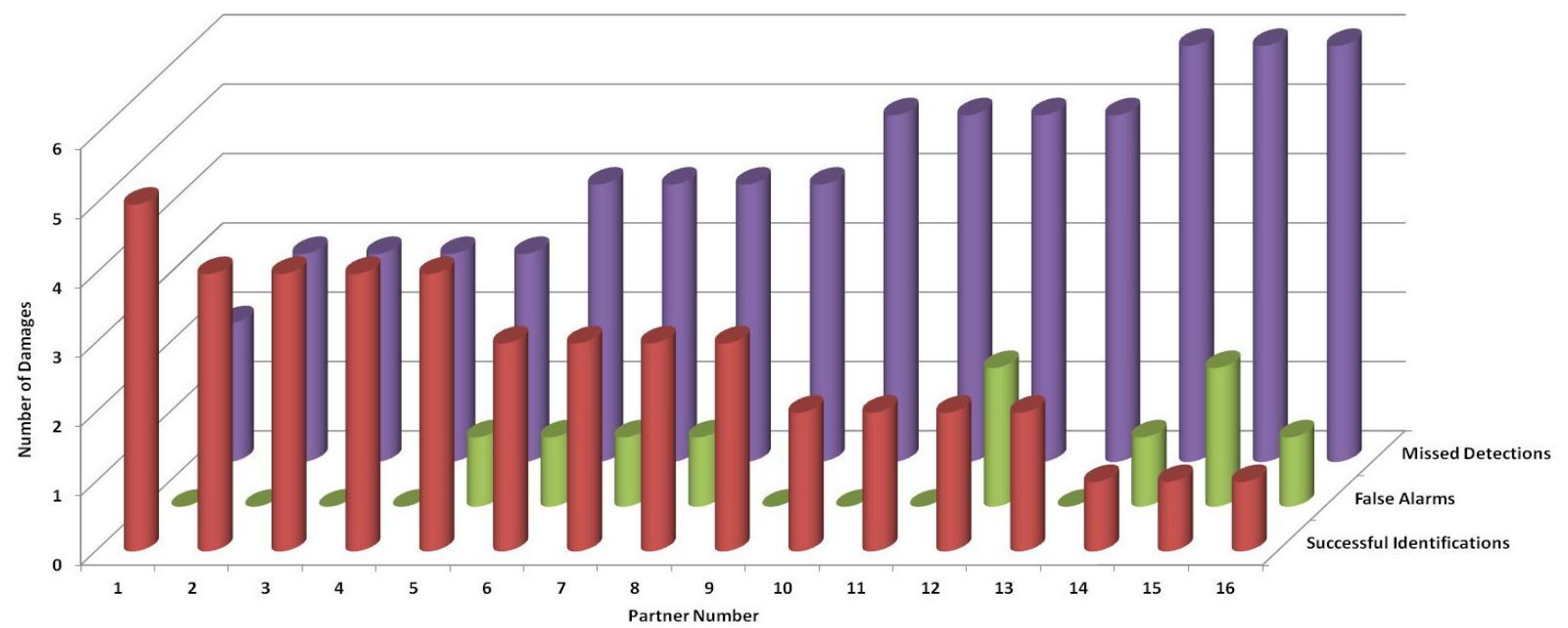

Figure 11. Comparison of damage results (Sheng, 2012)

The tabulated results were identified before receiving knowledge of the actual failure modes from NREL. The possible number of failures that can be identified from the vibration analysis for this Round Robin study is about seven, as listed in Table 5. The developed two-stage CM approach identified five failures and their severity levels. Moreover, the failures identified by the proposed vibration analysis approach do not have any false identification. The blind analysis results were submitted to NREL's gearbox Round Robin competition. A comparison of the results is shown in Figure 11. In the result comparison chart, this approach is masked as partner 1, which successfully detected five faults with accurate severity levels without producing any false alarm in the blind analysis. Further details on the NREL gearbox Round Robin study can be found in Sheng (2012).

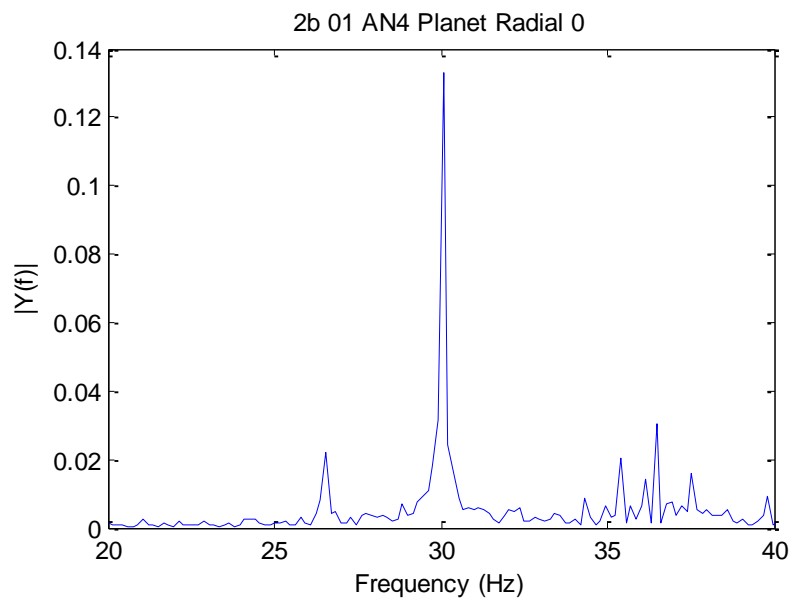

Figure 12. Damage 6, planet gear / sun pinion

After the blind analysis, the damage results were provided by NREL. The defects that were not identified in the blind analysis are defects in the upwind planet carrier and annulus/sun pinion. These defects are verified graphically, and the defect of the planet gear/sun pinion is identified with low severity as shown in Figure 12 (due to the high maximum amplitude at the desired frequency and the rise of the sidebands around the desired frequency), but the upwind planet carrier bearing defect was not identified in Figure 13. Please note that the upwind planet carrier bearing defect was not detected by the proposed method, as the damage was actually found to be on the non-running surface of the bearing.

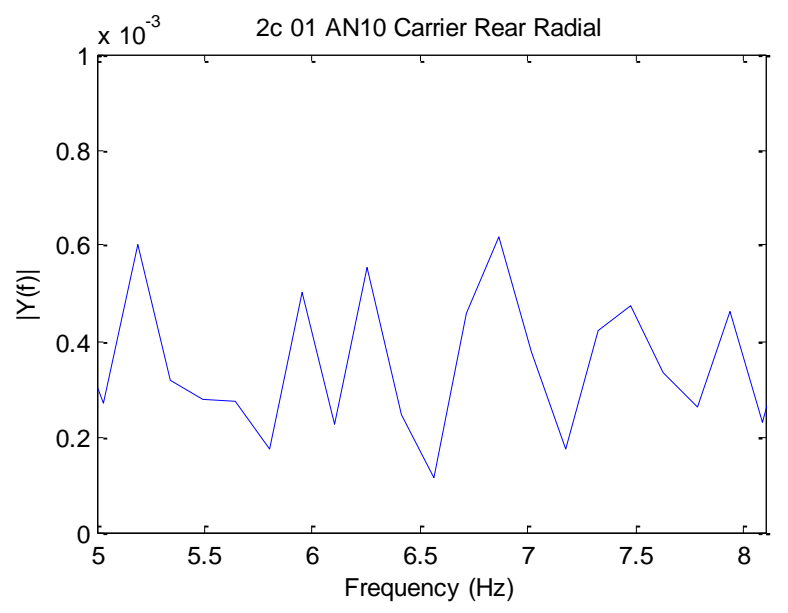

Figure 13. Damage 7, planet carrier UWB BPFO

\begin{tabular}{c|c|c|c}
\hline Damage & Component & Mode & Severity \\
\hline $\mathbf{6}$ & $\begin{array}{c}\text { Planet gear / } \\
\text { sun pinion }\end{array}$ & $\begin{array}{c}\text { Early stages of } \\
\text { gear failure }\end{array}$ & Low \\
\hline
\end{tabular}

Table 11. Additional faults identified during post-analysis

The additional defect component detected during the postresult analysis has been listed in Table 11, denoted as damage 6 in the damage analysis report. Because the desired 
frequencies of the upwind and downwind planet bearings and upwind and downwind planet carriers are less than 10 $\mathrm{Hz}$, the sidebands of these desired frequencies could most likely be overlapped with each other. Therefore, it is not quite effective to trace upwind planet carrier defects analytically or graphically.

\section{Conclusion}

This research showed that the developed vibration-based two-stage fault detection framework integrating both analytical diagnosis and graphical diagnosis is quite effective for analyzing gear and bearing faults in wind turbine transmissions, as demonstrated by the CM Round Robin study results. The proposed methodology detected the most faults (five out of seven) with correct severity levels in the blind analysis, and more importantly, did not produce any false alarm. Moreover, the post-result analysis was able to identify one more fault. The presented study was carried out without the use of any baseline information. The proposed approach could be further improved and verified using healthy gearbox testing data that NREL is going to provide as baseline data.

During the study, we found that it is quite useful to identify an initial set of potential failure modes using the analytical diagnosis method, which will substantially reduce the workload in processing massive high-frequency vibration data. With the preliminary results from the analytical diagnosis method, the graphical verification can be extremely useful to ensure correct diagnosis and avoid potential false identifications.

With the lessons learned from this CM Round Robin study, research on wind turbine condition monitoring can be further extended to failure prognostics through continuous $\mathrm{CM}$ and failure prediction, which will ultimately lead to automation of the wind farm maintenance decision-making process to reduce costs. Further study could also focus on developing a complex system design framework that can leverage the results of this study to quantify the functionality, reliability, and cost benefits of CM techniques and integrate them into a system-level wind turbine design practice, which may serve a fundamental solution of enhancing reliability and reducing wind turbine life-cycle cost.

\section{ACKNOWLEDGEMENT}

This research is partially supported by the National Science Foundation (CMMI-1200597) and the Kansas NSF EPSCoR program. The work conducted by National Renewable Energy Laboratory was supported by the U.S. Department of Energy under Contract No. DE-AC36-08-GO28308.

\section{REFERENCES}

Antoni, J. (2002). Differential diagnosis of gear and bearing faults. Journal of Vibration and Acoustics, 124 (2), pp. 165-171.

Byon, E., Perez, E., Ntaimo, L., \& Ding, Y. (2010). Simulation of wind farm operations and maintenance using DEVS. Simulation, pp. 1-25.

Ebeling, C. E. (1997). An introduction to reliability and maintainability engineering. Long Grove, IL: Waveland.

Errichello, R., \& Muller, J. (2012). Gearbox Reliability Collaborative gearbox 1 failure analysis report. NREL/SR-5000-53062. Golden, CO: National Renewable Energy Laboratory.

Gebraeel, N., Lawley, M., \& Liu, R. (2002). Vibrationbased condition monitoring of thrust bearings for maintenance management. Intelligent Engineering System Through Artificial Neural Network, 12, pp. 543551.

Harris, T.A. (2001). Rolling bearing analysis. 4th edition. New York, NY: John Wiley \& Sons, pp. 993-1000.

Lebold, M., McClintic, K.., Campbell, R., Byington, C., \& Maynard, K. (2000). Review of vibration analysis methods for gearbox diagnostics and prognostics. Proceedings of the 54th Meeting of the Society for Machinery Failure Prevention Technology, May 1-4, Virginia Beach, VA, USA.

Lu, W., \& Chu, F. (2010). Condition monitoring and fault diagnostics of wind turbines. Proceedings of Prognostics and Health Management Conference, pp. $1-11$.

McFadden, P.D., \& Smith, J.D. (1984). Vibration monitoring of rolling element bearings by the highfrequency resonance technique-a review. Tribology International, 17 (1), pp. 3-10.

Nielsen, J., \& Sorensen, J. (2010). On risk-based operation and maintenance of offshore wind turbine components. Reliability Engineering and System Safety, 96 (2011), pp. 218-229.

Nilsson, J., \& Bertling, L. (2007). Maintenance management of wind power systems using condition monitoring systems - Life cycle cost analysis for two case studies. IEEE Transactions on Energy Conversion, 22(1), pp. 223-229.

Randall, R.B. (2011). Vibration-based condition monitoring. Hoboken, NJ: Wiley.

Randall, R.B., \& Antoni, J. (2011). Rolling element bearing diagnostics - A tutorial. Mechanical Systems and Signal Processing, 25 (2), pp. 485-520.

Shi, W., Wang, F., Zhuo, Y., \& Liu, Y. (2010). Research on operation condition classification method for vibration monitoring of wind turbine. Proceedings of Power and Energy Engineering Conference APPEEC, pp. 1-6, Asia Pacific.

Sheng, S. (2012). Wind turbine gearbox condition monitoring round robin study - Vibration analysis. NREL/TP-5000-54530. Golden, CO: National Renewable Energy Laboratory. 
Sheng, S., Link, L., LaCava, W., van Dam, J., McNiff, B., Veers, P., Keller, J., Butterfield, S., \& Oyague, F. (2011). Wind turbine drivetrain condition monitoring during GRC phase 1 and phase 2 testing. NREL/TP5000-52748. Golden, CO: National Renewable Energy Laboratory.

SpectraQuest Tech Note. (2006). Analyzing gearbox degradation using time-frequency signature analysis.

Tamilselvan, P., Wang, P., \& Twomey, J. (2012). Quantification of economic and environmental benefits for prognosis informed wind farm operation and maintenance. $62^{\text {nd }}$ Annual IIE Industrial Systems and Engineering Research Conference (ISERC 2012), Orlando, FL, USA.

Tamilselvan, P., Wang, Y., \& Wang, P. (2012). Optimization of wind turbines operation and maintenance using failure prognosis. IEEE 2012 Prognostics and Health Management (PHM 2012), Denver, CO, USA.

Tamilselvan, P., Wang, P., \& Jayaraman, R. (2012). Diagnostics with unexampled faulty states using a twofold classification method. 2012 IEEE International Conference on Prognostics and Health Management, June 18-21, Denver, CO, USA.

Tamilselvan, P., \& Wang P. (2012). A hybrid inference approach for health diagnostics with unexampled faulty states. AIAA 2012-1784, 53 ${ }^{\text {rd }}$ AIAA/ASME/ASCE /AHS/ASC Structures, Structural Dynamics, and Materials Conference, April 23-26, Honolulu, Hawaii, USA.

Tamilselvan, P., \& Wang, P. (2013). Failure diagnosis using deep belief learning based health state classification. Reliability Engineering \& System Safety, 115 (2013), pp. 124-135.

Yang, W., Tavner, P., \& Wilkinson, M. (2008). Wind turbine condition monitoring and fault diagnosis using both mechanical and electrical signatures. Proceedings of IEEE International Conference on Advanced Intelligent Mechatronics, pp. 1296-1301.

\section{BIOGRAPHIES}

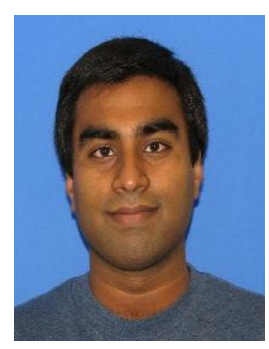

Prasanna Tamilselvan received his bachelor's degree in mechanical engineering in 2007 from Anna University in India and his master's degree in 2010 in industrial and manufacturing engineering from Wichita State University. $\mathrm{He}$ is currently pursuing his Ph.D. in the industrial and manufacturing engineering department at Wichita State University. He received the IEEE Best Paper Award on Prognostics and Health Management (PHM) in 2012. His expertise is in the areas of machine learning applications in failure diagnostics and prognostics.

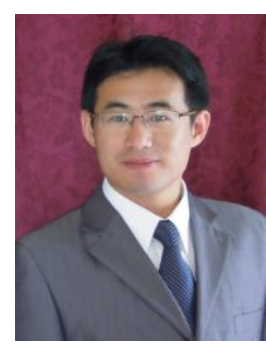

Dr. Pingfeng Wang is currently an assistant professor in the industrial and manufacturing engineering department at Wichita State University. Dr. Wang got his bachelor's degree in mechanical engineering in 2001 from The University of Sci. \& Tech. in Beijing, China; his master's degree in 2006 in applied mathematics from Tsinghua University in China; and his Ph.D. in mechanical engineering from the University of Maryland at College Park in 2010. His expertise is in the areas of reliability analysis and design for complex systems, structural health prognostics, and condition-based maintenance. He has written over 50 research articles in these areas, including one ASME Best Paper Award in 2008 and IEEE Best Paper Award in 2012. Since joining Wichita State University in 2010, he has focused his research on developing methodologies to integrate reliability-based complex system design with health prognostics to improve the overall system resilience and sustainability.

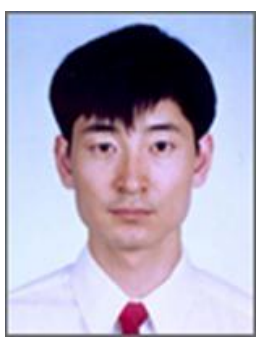

Dr. Shuangwen (Shawn) Sheng is a senior engineer at the National Renewable Energy Laboratory (NREL). He has B.S. and M.S. degrees in electrical engineering and a Ph.D. in mechanical engineering. Shawn is currently leading wind turbine condition monitoring, failure database, and wind plant O\&M research at NREL. Shawn also has experience in mechanical and electrical system modeling and analysis, soft computing techniques, and automatic control. He has published his work in various journals, conference proceedings, and book chapters.

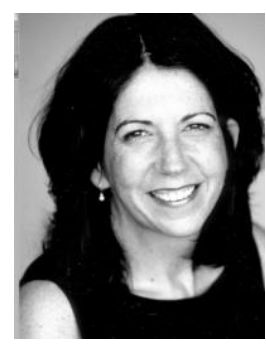

Dr. Janet M. Twomey received her B.S., M.S. and Ph.D. in industrial engineering from the University of Pittsburgh. Dr. Twomey is currently a professor of industrial and manufacturing engineering at Wichita State University. From 2001 to 2004, she held the position of program officer for Manufacturing Enterprise Systems at the National Science Foundation (NSF). Prior to going to NSF, her research was in computational intelligence and sparse data. For this work she received an NSF CAREER Award. Dr. Twomey's current research applies life-cycle thinking to analyze energy use in the industrial and commercial sectors. 